\title{
LIMITS OF SEQUENCES OF FINITELY GENERATED ABELIAN GROUPS
}

PAUL HILL ${ }^{1}$

1. Introduction. The limits referred to in the title are the ordinary direct and inverse limits, but for our purpose we shall require throughout $\S \S 2$ and 3 that the homomorphisms associated with direct limits be monomorphisms and that those associated with inverse limits be epimorphisms. Denote by $\mathfrak{C}_{D}\left(\mathfrak{C}_{I}\right)$ the collection of sequences of groups for which a direct (inverse) limit exists and is determined up to isomorphism solely by the groups, that is, is independent of the choice of the monomorphisms (epimorphisms). In $\$ \S 2$ and 3, respectively, the sequences of finitely generated abelian groups belonging to $\mathfrak{C}_{D}$ and $\mathfrak{C}_{I}$ are determined. A characterization of inverse limits of sequences of finitely generated abelian groups is given in $\$ 4$.

Since our attention will be restricted to abelian groups, "abelian group" is abbreviated to "group". With one exception, all groups are considered discrete.

2. Direct limits. We wish to consider only sequences $G_{n}$ which admit monomorphisms from $G_{n}$ to $G_{n+1}$; such sequences will be called ascending. A basis $\left[g_{1}, g_{2}, \cdots, g_{k}\right]$ of a finite primary group is said to be written in canonical form if its elements are arranged in decreasing order, $O\left(g_{i+1}\right) \leqq O\left(g_{i}\right)$ for $1 \leqq i<k$ where $O(x)$ denotes the order of $x$. Let $G_{n}$ be an ascending sequence of finite $p$-primary groups with canonical bases $\left[g_{1, n}, g_{2, n}, \cdots, g_{k(n), n}\right]$. Notice that the sequences $k(n)$ and $O\left(g_{i, n}\right)$ (with $i$ fixed) are monotonic since $G_{n}$ is ascending. The rank (finite or $\infty$ ) of the sequence $G_{n}$ is defined as $r=\lim _{n \rightarrow \infty} k(n)$, and for each natural integer $i \leqq r, m_{i}$ is defined by $p^{m_{i}}=\lim _{n \rightarrow \infty} O\left(g_{i, n}\right)$. Set $m=\min \left\{m_{i}\right\}$.

The sequence $G_{n}$ is called irregular if for some fixed $m_{0}<m$ the relation $O\left(g_{i, n}\right)=p^{m_{0}}$ holds for almost all $n$ with a suitable choice of $i$, that is, $G_{n}$ is irregular if all but a finite number of the groups $G_{n}$ contain a summand of the same order $p^{m} 0<p^{m}$. Sequences are said to be regular if they are not irregular. Obviously, if the rank of the sequence $G_{n}$ is finite, then $G_{n}$ is regular.

THEOREM 1. A necessary and sufficient condition for an ascending sequence $G_{n}$ of finite primary groups to belong to $\mathcal{C}_{D}$ is that $G_{n}$ be regular.

Proof. The fact that the condition is necessary is almost trivial.

Received by the editors November 2, 1960.

1 A National Science Foundation postdoctoral fellow. 
The most natural ${ }^{2}$ direct limit of $G_{n}$ is $A=\sum \oplus C\left(p^{m_{i}}\right)$, where $C\left(p^{m_{i}}\right)$ is the cyclic group of order $p^{m_{i}}$ for finite $m_{i}$ and $C\left(p^{\infty}\right)$ is quasicyclic (=group of type $p^{\infty}$ ). However, if $G_{n}$ is irregular, almost all the groups $G_{n}$ contain a cyclic summand (isomorphic to) $C\left(p^{m_{0}}\right)$ where $m_{0}<m$; clearly, this summand can be preserved (as a summand) in some direct limit. Since such a limit cannot be isomorphic to $A, G_{n} \in \mathbb{C}_{D}$.

In order to prove that regularity is sufficient, let $G_{n}$ be regular and let $B$ be an arbitrary direct limit of $G_{n}$. Use is made of the limit $A$ defined above; we show that $B \cong A$. Recall that a direct limit $L$ of $G_{n}$ (under monomorphisms) is the set-theoretical union of groups isomorphic to the $G_{n} ; L=\bigcup H_{n}$ where $H_{n} \cong G_{n}$ and $H_{n} \subseteq H_{n+1}$. Thus it is immediate (whether $G_{n}$ is regular or not) that the rank (for definition, see [1]) of $B$ is precisely the rank of the sequence $G_{n}, r(B)=r$.

If $m=\infty$, then $B$ is divisible; for otherwise $B$ would have a cyclic summand $C(\neq 0)$, which would imply that almost all the $G_{n}$ contained a summand isomorphic to $C$. Since $G_{n}$ is regular, this is impossible. Thus $B \cong A$ since both are divisible with the same rank, and the theorem is proved in the case $m=\infty$.

For the case $m<\infty$, let $B=B_{R} \oplus B_{D}$ where $B_{R}$ is reduced and $B_{D}$ is divisible. $B_{R}$ is bounded since $B_{R}$ unbounded implies that $B_{R}$ $=C_{1} \oplus C_{2} \oplus \cdots \oplus C_{k} \oplus B_{R}^{\prime}$, where $k$ and the orders of the cyclic groups $C_{i}$ are arbitrarily large; but this implies $m=\infty$. Hence $B_{R}$ is a direct sum of cyclic groups, and $B=B_{1} \oplus B_{2} \oplus \cdots \oplus B_{D}$ where $B_{i}$ is a direct sum of cyclic groups of order $p^{i}$. Since $G_{n}$ is regular, $B_{1}=B_{2}=\cdots=B_{m-1}=0$. Using the corresponding notation, we write $A=A_{m} \oplus A_{m+1} \oplus \cdots \oplus A_{D}$.

It is immediate that $p^{t} B(t \geqq 0)$ is a direct limit of the sequence $p^{t} G_{n}$; hence the rank of $p^{t} B$ is determined solely by the groups $G_{n}$. Thus $A_{D} \cong B_{D}$ since the reduced parts of $A$ and $B$ are bounded. Moreover, $r\left(p^{m} B\right)=r\left(p^{m} A\right)<\infty$, and

$$
r\left(A_{m}\right)=r(A)-r\left(p^{m} A\right)=r(B)-r\left(p^{m} B\right)=r\left(B_{m}\right) ;
$$

hence $A_{m} \cong B_{m}$. Similarly,

$$
r\left(A_{m+1}\right)=r\left(p^{m} B\right)-r\left(p^{m+1} B\right)=r\left(B_{m+1}\right) ;
$$

$A_{m+1} \cong B_{m+1}$, etc.

Since any subgroup $\neq 0$ of the additive rationals is a direct limit of infinite cyclic groups, we have at once

Corollary 1. An ascending sequence $G_{n}$ of finitely generated groups belongs to $\mathcal{C}_{D}$ if and only if the groups $G_{n}$ are finite and the p-primary components form a regular sequence for each prime $p$.

\footnotetext{
${ }^{2}$ The limit $A$ is obtained merely by identifying $g_{i, n}$ with a multiple of $g_{i, n+1}$.
} 
3. Inverse limits. Here, we wish to consider only sequences $G_{n}$ which admit epimorphisms from $G_{n+1}$ to $G_{n}$; such sequences will be called extending. However, a sequence of finite (abelian) groups is extending if and only if it is ascending; hence the terminology established in $\$ 2$ carries over to extending sequences of finite $p$-groups.

Theorem 2. A necessary and sufficient condition for an extending sequence $G_{n}$ of finite primary groups to belong to $\mathfrak{C}_{I}$ is that $G_{n}$ be regular.

Proof. Again, it is immediate that the condition is necessary. In fact, one needs only to make the obvious replacements in the proof of Theorem 1. Recall that $\sum \oplus$, the direct sum, should be replaced by $\sum^{\prime} \oplus$, the complete direct sum, and the group of type $p^{\infty}$ by the $p$-adic group.

Denote by $G^{*}$ the (discrete) character group of $G$. With an inverse sequence

$$
G_{1} \leftarrow G_{2} \leftarrow \cdots \leftarrow G_{n} \leftarrow \cdots
$$

there is associated in a natural way a (unique) direct sequence

$$
G_{1}^{*} \rightarrow G_{2}^{*} \rightarrow \cdots \rightarrow G_{n}^{*} \rightarrow \cdots,
$$

which in turn gives rise to an inverse sequence

$$
G_{1}^{* *} \leftarrow G_{2}^{* *} \leftarrow \cdots \leftarrow G_{n}^{* *} \leftarrow \cdots
$$

Together with the usual isomorphism (see, for example, [2]) between $G_{n}$ and $G_{n}^{* *},(1)$ and (3) form a commutative diagram and therefore have isomorphic limits. However, the limit of (3) is the character group of the limit of (2). Thus if $G_{n}$ is regular, by Theorem 1 the limit of (1) is determined by the groups alone and $G_{n} \in \mathfrak{C}_{I}$.

Now let $G_{n}$ be an extending sequence of finitely generated groups. Analogous to the $p$-rank of a sequence, the torsion free rank of the sequence $G_{n}$ is defined as the limit of the torsion free ranks of the groups $G_{n}, r_{0}=\lim _{n \rightarrow \infty} r_{0}\left(G_{n}\right)$. The question of whether or not $G_{n}$ is contained in $\mathfrak{C}_{I}$ depends to a large degree on whether $r_{0}$ is finite or not; consequently, the two cases are distinguished.

CoROllaRY 2. Let $G_{n}$ be an extending sequence with finite torsion free rank of finitely generated groups. $A$ necessary and sufficient condition for $G_{n}$ to belong to $\mathfrak{C}_{I}$ is that the p-primary components form a regular sequence for each prime $p$.

Proof. Denoting the torsion free rank of the sequence $G_{n}$ by $r_{0}$, we may as well assume that the torsion free rank of the group $G_{n}$ is $r_{0}$ for each $n$. Clearly, an inverse limit of $G_{n}$ is the free group of rank 
$r_{0}$ plus a limit of the torsion subgroups of $G_{n}$. The proof is completed by Theorem 2 .

Corollary 3. Let $G_{n}$ be an extending sequence with infinite torsion free rank of finitely generated groups. A necessary and sufficient condition for $G_{n}$ to belong to $\mathfrak{C}_{I}$ is that the torsion subgroups of $G_{n}$ do not contribute to any inverse limit of $G_{n}$, that is, there is no nonzero limit element $\left\{x_{n}\right\}$ where $x_{n}$ is torsion for all $n$.

Proof. It is clear that the complete direct sum of a countably infinite number of infinite cyclic groups is an inverse limit of $G_{n}$; denote this group by $A$. Let $B$ be an arbitrary inverse limit of $G_{n}$ and let $C$ denote the subgroup of $B$ which comes from the torsion subgroups, $T_{n}$, of $G_{n}$. Then $B / C$, isomorphic to an inverse limit of $G_{n} / T_{n}$ (since the $T_{n}$ are finite), is isomorphic to $A$. Hence if $C=0$, then $B \cong A$; but if $C \neq 0$, then it contains either a cyclic group of prime order or a $p$-adic group, so $B$ is not isomorphic to $A$.

4. Totality of limits. The requirement that the homomorphisms associated with direct and inverse limits be monomorphisms and epimorphisms, respectively, is dropped. It is immediate that the direct limits of sequences of finitely generated groups are just the countable groups. The class of inverse limits of such sequences is given by the following

TheOREM 3. A necessary and sufficient condition for a group $G$ to be an inverse limit of a sequence of finitely generated (abelian) groups is that $G$ be the complete direct sum of (at most) a countable number of cyclic and p-adic groups.

Proof. It is obvious that the condition is sufficient. In order to prove that it is necessary, let $G$ be an inverse limit of a sequence $G_{n}$ of finitely generated groups and let $C$ denote the subgroup of $G$ which comes from the torsion subgroups, $T_{n}$, of $G_{n}$. The factor group $G / C$, isomorphic to an inverse limit of $G_{n} / T_{n}$, is the complete direct sum of a countable number of infinite cyclic groups. It is well known that $C$ admits a compact topology in which it is totally disconnected and satisfies the second axiom of countability. Since the Pontrjagin dual group $C^{*}$ of $C$ is countable, it follows from the proof of Proposition 3.1 of [3] that $C$ is a complete direct sum of a countable number of cyclic and $p$-adic groups. Since $C$ is compact, it is algebraically compact [4]; hence its purity implies that it is a direct summand of $G$, and the theorem is proved.

5. Remark and example. In conclusion we add the following remark relevant to $\$ \S 2$ and 3 . There are direct (inverse) sequences, 
even of finite groups, which have two nonisomorphic limits associated with monomorphisms (epimorphisms) $\rho_{n}$ and $\phi_{n}$, respectively, such that $\rho_{n} \neq \phi_{n}$ only if both $\rho_{n}$ and $\phi_{n}$ are onto isomorphisms. In fact, let $G$ be a countable, reduced $p$-primary group with (nonzero) elements of infinite height; an increasing sequence of finite subgroups $G_{n}$ which leads up to $G$ exhibits such a sequence if the $G_{n}$ are chosen such that the index of $G_{n}$ in $G_{n+1}$ is 0 or $p$, depending on whether $n$ is odd or even.

\section{REFERENCES}

1. L. Fuchs, Abelian groups, Budapest, Publishing House of the Hungarian Academy of Sciences, 1958.

2. M. Hall, The theory of groups, New York, The Macmillan Company, 1959.

3. D. K. Harrison, Infinite abelian groups and homological methods, Ann. of Math. vol. 69 (1959) pp. 366-391.

4. J. Los, Abelian groups that are direct summands of every abelian group which contains them as pure subgroups, Fund. Math. vol. 44 (1957) pp. 84-90.

INSTITUTE FOR ADVANCED STUdY 